\title{
Predicting Depression and Anxiety Among Adults with Disabilities During the COVID-19 Pandemic
}

\author{
Katie Wang ${ }^{1}$, Robert B. Manning III ${ }^{1}$, Kathleen R. Bogart ${ }^{2}$, Jonathan M. Adler ${ }^{3}$, Michelle R. \\ Nario-Redmond ${ }^{4}$, Joan M. Ostrove ${ }^{5}$, Sarah R. Lowe ${ }^{1}$ \\ ${ }^{1}$ Department of Social and Behavioral Sciences, Yale School of Public Health \\ ${ }^{2}$ School of Psychological Science, Oregon State University \\ ${ }^{3}$ Olin College of Engineering \\ ${ }^{4}$ Departments of Psychology and Biohumanities, Hiram College \\ ${ }^{5}$ Department of Psychology, Macalester College. \\ This manuscript is in press at Rehabilitation Psychology.
}

This research was supported by funding from the Yale School of Public Health COVID-19 Rapid Response Pilot Gift awarded to Dr. Wang.

Correspondence concerning this article should be addressed to Katie Wang, Department of Social and Behavioral Sciences, Yale School of Public Health, 60 College Street, Suite 400, New Haven CT 06510. Email: katie.wang@yale.edu 


\begin{abstract}
Purpose/Objective: Emerging research has highlighted sources of magnified stress and trauma for people with disabilities during the COVID-19 pandemic, as compared to others in the general population. However, little research has examined the mental health impact of the pandemic on people with disabilities in relation to disability-related stigma, social isolation, and demographic characteristics. The present study therefore sought to identify predictors of depression and anxiety symptoms among U.S. adults with disabilities during the COVID-19 pandemic.
\end{abstract}

Research Methods/Designs: Data were collected online between October and December 2020. U.S. adults with disabilities $(n=441)$ completed self-report measures of depression, anxiety, psychosocial processes, and a range of demographic and disability characteristics.

Results: In our sample, $61.0 \%$ and $50.0 \%$ of participants met criteria for a probable diagnosis of major depressive disorder and generalized anxiety disorder, respectively. Participants also experienced significantly higher levels of disability-related stigma and social isolation compared to pre-pandemic norms. Hierarchical regression analyses identified higher social isolation, presence of chronic pain, younger age, higher disability-related stigma, and higher worries about contracting COVID-19 as significant predictors of both depression and anxiety symptoms.

Conclusion/implications: This study highlights important demographic and psychosocial predictors of mental health risks for people with disabilities in the context of COVID-19. Findings further underscore the need to attend to those at elevated risk within the disability community as rehabilitation professionals, disability organizations, and policymakers work to support people with disabilities in post-pandemic recovery and create a more equitable response to ongoing and future public health crises. 


\section{Predicting Depression and Anxiety Among Adults with Disabilities During the COVID-19}

\section{Pandemic}

- In a large study of people with disabilities during the COVID-19 pandemic, we found high levels of depression and anxiety. Accessible and disability culturally competent mental and physical healthcare is crucial and may be provided through a number of modalities, including telehealth or home visits.

- Social isolation was the strongest predictor of depression and anxiety. Virtual disability communities, support groups, and peer support are useful avenues for providing social connection during public health crises.

- Stigma and worries about COVID-19 were also associated with depression and anxiety. These findings point to the urgent need for public health policy to address systemic ableism and to increase accessibility of emerging public health information in a variety of formats.

The novel coronavirus disease 2019 (COVID-19) has taken an unprecedented toll on the health and well-being of people and communities worldwide. As of August 2021, COVID-19 has led to the deaths of more than four million since its onset in March 2020 (Centers for Disease Control and Prevention, 2021). In addition to eliciting fear of viral transmission and grief surrounding the overwhelming loss of human life, the COVID-19 pandemic has necessitated myriad viral containment measures (e.g., stay-at-home orders, physical distancing), which contributed to pervasive unemployment and financial loss (Bureau of Labor Statistics, 2020), as well as social isolation and loneliness (Hwang et al., 2020). This confluence of psychosocial stressors has been particularly salient for people with disabilities, who, despite comprising $25 \%$ 
of the U.S. population (Okoro et al., 2018), continue to experience systematic and individual ableism in the forms of stigma, interpersonal violence, and structural disadvantage (e.g., poverty, inadequate transportation, limited healthcare access; Nario-Redmond, 2019).

Emerging research has highlighted a number of additional stressors for people with disabilities, relative to others in the general population, including difficulties with accessing healthcare and rehabilitative services, difficulties obtaining basic supplies (e.g., groceries, medications), concerns about medical rationing, and disproportionate exposure to pandemicrelated adversity (e.g., social isolation, loss of employment and income; for reviews, see Andrews et al., 2020; Jesus et al., 2021; Lund et al., 2020). For example, according to a survey conducted in March and April of 2020 that included 2,469 American adults with disabilities, $44 \%$ of participants reported experiencing new challenges in obtaining non-COVID-related medical treatment due to the pandemic; of the $64 \%$ of participants who received routine health care, more than half reported that their care had been disrupted by the pandemic (Drum et al., 2020). Further, given that people with disabilities tended to have smaller social support networks and were twice as likely to be in poverty than their nondisabled counterparts even prior to the pandemic (Erickson et al., 2017; Tough et al., 2017), they are likely to be in a particularly precarious position in the face of the pandemic's pervasive social and economic impacts. Notably, a U.K. survey in March 2020 indicated that, compared to people without disabilities, those with disabilities reported more than double the rate of self-harm/suicidal thoughts (48.0\% vs. $17.8 \%)$ and more than triple the rate of self-harm behaviors (17.8\% vs. $4.9 \%$; Iob et al., 2020).

Despite these well-documented challenges and disparities, few empirical studies have examined the mental health impact of the pandemic on people with disabilities in relation to 
various demographic and psychosocial characteristics. This limitation is noteworthy given that the disability community is highly heterogeneous with diverse experiences and support needs. Identifying factors that exacerbate or mitigate mental health risks within this population will help inform the efforts of rehabilitation professionals, disability organizations, and policymakers as they work to support people with disabilities in post-pandemic recovery and create a more equitable response to ongoing and future public health crises. To this end, the present study examined how demographic and disability characteristics, COVID-19-related stressors, social isolation, and perceptions of disability-related stigma might be associated with depression and anxiety symptoms among a U.S. sample of adults with disabilities.

To our knowledge, only two survey studies to date have examined demographic and psychosocial predictors of mental health risks in the context of disability and COVID-19. In their online survey of 1,027 Canadian adults with disabilities conducted in June 2020, Pettinicchio et al. (2021) showed that worries about contracting COVID-19, financial hardship, and social isolation were significantly associated with increased stress, anxiety, and despair over the past two weeks. Additionally, in a survey of 269 U.S. adults with disabilities and chronic illnesses, Umucu and Lee (2020) found that, while controlling for depression, anxiety, and stress related to COVID-19, active coping, use of emotional support, humor, and religion were positively associated with psychological well-being, whereas self-blame was negatively associated with psychological well-being.

While these studies yielded valuable initial insights, they also highlight two areas that warrant further investigation. First, perceptions and experiences of disability-related stigma (i.e., devaluation and discrimination based on one's disability) have played an important role in driving many of the pandemic-related challenges and disparities facing people with disabilities 
(Andrews et al., 2020; Jesus et al., 2021; Lund et al., 2020), yet associations between mental health outcomes and disability-related stigma have not, to our knowledge, been empirically examined. Even prior to the pandemic, evidence from a representative European sample indicated that disability-related stigma was more deleterious to health and well-being compared to stigma associated with other socially disadvantaged identities (Branco et al., 2019). Second, in line with many large web-based surveys, Pettinicchio et al. (2021) utilized single-item, dichotomous measures to assess the presence (vs. absence) of increases in stress, anxiety, and despair. Studies that utilize multi-item, validated, and continuous measures of depression and anxiety symptoms, while also adjusting for mental health diagnoses present prior to the start of the pandemic, can provide more nuanced information about the impact of the pandemic on the full spectrum of mental health symptomatology among people with disabilities.

In light of these gaps in the literature, the present descriptive study extended prior work by explicitly considering the roles of disability-related stigma and social isolation, along with pandemic-specific stressors (e.g., financial loss, difficulty accessing basic supplies) and demographic characteristics, as potential predictors of depression and anxiety symptoms assessed via validated, continuous measures. Consistent with the extant literature, we hypothesized that disability-related stigma, social isolation, worries about contracting COVID19, difficulty accessing basic supplies, and financial loss would be significantly associated with depression and anxiety symptoms (Lund et al., 2020; Pettinicchio et al., 2021). Additionally, given that the COVID-19 pandemic is likely to be particularly stressful for people with disabilities with high support needs (Jesus et al., 2020), we hypothesized that those who identify as having multiple disabilities and experience higher levels of functional impairment (i.e., pain, low energy, and difficulty with completing daily activities) would experience higher levels of 
depression and anxiety. Lastly, consistent with research on the mental health impact of the COVID-19 pandemic in the general population (O'Connor et al., 2021; Qiu et al., 2020), we hypothesized that younger participants and participants with pre-pandemic mental health conditions would experience higher levels of depression and anxiety.

\section{Method}

\section{Participants and Procedures}

Data for the current study were taken from a larger online survey that examined the health and psychosocial impacts of the COVID-19 pandemic on U.S. adults with disabilities. Participants, all of whom self-identified as having a disability, were recruited from disabilityfocused social media platforms (e.g., Disability Wisdom Discussion Group on Facebook) and email listservs for disability organizations (e.g., Society for Disability Studies) between October and December 2020. Individuals reached through these channels were provided with a web link to the survey, which was administered via Qualtrics. Each participant received a $\$ 20$ gift card for completing the survey. All study measures were approved by the (masked for peer review) institutional review board. The final analytic sample consisted of 441 participants who responded to all questions within the survey.

\section{Measures}

\section{Disability Stigma}

The Stigma Scale for Chronic Illness - 8 (SSCI-8; Molina et al., 2013) is an 8-item selfreport measure of enacted and internalized stigma validated for use among individuals with a wide range of disabilities and chronic health conditions. Participants rated each item (e.g., "Because of my disability, some people avoid me", "I feel embarrassed about my disability) on a scale ranging from 1 (never) to 5 (always). This scale was scored using published $\mathrm{T}$ scores 
calibrated such that a mean of 50 and standard deviation of 10 are representative of the norms for populations with disabilities and chronic illnesses (National Institute of Neurological Disorders and Stroke, 2015). Higher scores indicate greater experiences with disability-related stigma. Cronbach's $\alpha$ in the current sample was .82.

\section{Social Isolation}

The Patient-Reported Outcomes Measurement Information System Short Form version 2.0 - Social Isolation 4a (PROMIS Short Form v2.0 - Social Isolation 4a; Hahn et al., 2014) is a 4-item self-report measure that assesses perceptions of being alone, disconnected, avoided, or excluded by others (e.g., "I feel that people barely know me.”) Participants rated each item on a scale ranging from 1 (never) to 5 (always). The scale was scored using the published $\mathrm{T}$ scores calibrated such that a mean of 50 and standard deviation of 10 are representative of the general U.S. population norms. Higher scores indicate greater perceptions of social isolation. Cronbach's $\alpha$ in the current sample was .80.

\section{Major Depressive Disorder (MDD) Symptoms}

The Patient Health Questionnaire - 9 (PHQ-9; Kroenke et al., 2001) is a 9-item selfreport measure assessing depressive symptoms over the past two weeks. Participants rated each item on a four-point scale ranging from 0 (not at all) to 3 (nearly every day); items were

summed, with higher scores indicating higher levels of depressive symptoms. Scores of 10 or higher indicate depressive symptoms consistent with a diagnosis of major depressive disorder (Kroenke et al., 2001). Cronbach's $\alpha$ in the current sample was .86.

\section{Anxiety Symptoms}

The Generalized Anxiety Disorder - 7 (GAD-7; Spitzer et al., 2006) is a 7-item selfreport measure assessing anxiety symptoms over the past two weeks. Participants rated each item 
on a four-point scale ranging from 0 (not at all) to 3 (nearly every day). Items were summed, with higher scores indicating higher levels of anxiety symptoms. Scores of 10 or higher indicate anxiety symptoms consistent with a diagnosis of generalized anxiety disorder (Spitzer et al., 2006). Cronbach's $\alpha$ in the current sample was 86 .

\section{Worries about COVID-19}

Worries about COVID-19 were assessed using two items taken from the COVID-19 Community Survey Question Bank (CDC, 2020): "How worried are you that you might get seriously ill or die due to COVID-19?" and "How worried are you that a family member or close friend might get seriously ill or die due to COVID-19?" Participants rated each item on a 5-point scale ranging from 1 (extremely worried) to 5 (not at all worried). Items were reverse coded and averaged, with higher scores indicating higher levels of worries about COVID-19.

\section{Financial Loss, Lack of Basic supplies, and COVID-19 Exposure}

Participants indicated whether they have experienced any of the following since the start of the COVID-19 pandemic: (1) personal financial loss (e.g., lost wages, job loss, investment/retirement loss, travel-related cancelations); (2) not having enough basic supplies (e.g., food, water, medications, a place to stay); (3) having had COVID-19; and (4) having lost a family member or close friend to COVID-19 (CDC, 2020; Harkness et al., 2020). Participants responded "yes" or "no" to each item; for the item assessing COVID-19 status, an additional response option of "probably yes/I think so" was included to account for the scarcity of diagnostic testing early in the pandemic. A dichotomous variable was created for each item, with "yes" coded as 1 and "no" coded as 0. For the item assessing COVID-19 status, the "probably yes/I think so" response option was also coded as 1.

\section{Covariates}


Pre-pandemic mental health conditions. Participants were presented with a list of mental health conditions (i.e., depression, anxiety, panic disorder, obsessive-compulsive disorder, substance use disorder, posttraumatic stress disorder, attention deficit hyperactivity disorder, other) and asked to indicate whether they have been diagnosed with each condition prior to the start of the COVID-19 pandemic. A dichotomous variable was created, such that participants who selected any of the mental health diagnoses received a score of 1 and the rest received a score of 0 .

Functional Impairment. To assess participants' level of functional impairment, we included three items from the Physical Health subscale of the World Health Organization Quality of Life - Brief form (WHOQOL-Brief; WHO, 1998) to capture experiences of physical pain, energy levels, and ability to complete daily activities. Pain was assessed using the item "To what extent do you feel that physical pain prevents you from doing what you need to do?", rated on a five-point scale from 1 (not at all) to 5 (an extreme amount). Energy level was assessed using the item "Do you have enough energy for everyday life?", rated on a five-point scale from 1 (not at all) to 5 (completely). Ability to complete daily activities was assessed using the item "How satisfied are you with your ability to perform your daily living activities?", rated on a five-point scale from 1 (very dissatisfied) to 5 (very satisfied).

\section{Data Analysis}

Prior to analyses to fulfill our study aims, a series of preliminary analyses were conducted. First, as recommended by Tabachnick and Fidell (2007), all study variables were assessed for assumptions of normality. Next, descriptive statistics (i.e., means, standard deviations, frequencies) were calculated for all study variables. Given the small number of participants in some of the demographic categories, the education, race, and employment 
variables were recoded into variables of less than a bachelor's degree versus a bachelor's degree or higher, White versus non-White, and employed (full- or part-time) versus unemployed. Following this, bivariate correlations among all study variables were calculated.

We then assessed prevalence of probable major depressive disorder and generalized anxiety disorder in our sample using established cutoff scores for PHQ-9 (Kroenke et al., 2001) and GAD-7 (Spitzer et al., 2006). Stigma and social isolation levels were compared to pre-pandemic norms using one sample t-tests.

Subsequently, two hierarchical linear regression models were estimated to predict depression and anxiety symptoms. In Step 1 of each model, demographic and disability characteristics that have been shown to predict mental health in the context of disability and/or COVID-19 (Hennein et al., 2021; Pettinicchio et al., 2021) were entered. These include gender, race, ethnicity, employment status, education, having a pre-pandemic mental health condition, and having multiple disabilities. In Step 2 of each model, worries about COVID-19, along with other pandemic-related stressors (i.e., financial loss, lack of basic supplies, COVID-19 exposure and bereavement) were entered. In Step 3 of each model, social factors hypothesized to predict depression and anxiety symptoms, including disability-related stigma and social isolation, were entered.

\section{Results}

\section{Preliminary Analyses}

Tables 1 and 2 summarize the sample's demographic and disability characteristics. As shown, the mean age of participants was 35 years $(S D=10.04) ; 50.9 \%(n=227)$ identified as female, and $71.2 \%(n=317)$ identified as White. On average, participants had been living with their disability for 17 years $(S D=13.77)$. Nearly half $(48.9 \%, n=216)$ of the participants 
identified as having a physical/mobility disability, followed by emotional/behavioral disorder $(31.8 \%, n=142)$ and other health impairment $(26.7 \%, n=119) ; 43.7 \%(n=195)$ identified as having more than one disability. Over half $(65.9 \% ; n=294)$ of participants indicated being diagnosed with a mental health condition prior to the COVID-19 pandemic.

In our sample, 272 (61.0\%) participants met the screening cutoff for major depressive disorder, and 223 (50.0\%) met the screening cutoff for generalized anxiety disorder. One sample $t$-tests found that our participants experienced significantly higher levels of stigma, $t(445)=$ $37.39, p<.001$, and social isolation, $t(445)=15.12, p<.001$, compared to published prepandemic norms in a disabled and chronically ill sample (National Institute of Neurological Disorders and Stroke, 2015) and a general population sample (Hahn et al., 2014), respectively.

See Table 3 for bivariate correlations. In order to speak to the gap in the literature around social factors, correlations with stigma and social isolation are highlighted here. Disability stigma was more likely among those identified as Hispanic or Latino, unemployed, male gender, or having multiple disabilities, pre-pandemic mental health conditions, physical pain, lower energy, lower satisfaction with performance of daily living activities, social isolation, depression, anxiety or not having enough basic supplies. Social isolation was more likely among those who were younger, unemployed, trans/nonbinary, or having multiple disabilities, pre-pandemic mental health conditions, pain, lower energy, lower satisfaction with performance of daily living activities, having experienced COVID-19, disability stigma, depression, anxiety, or not having enough basic supplies.

\section{Hierarchical Regression Analyses}


Table 4 shows the results of the hierarchical regression models predicting first depression and then anxiety symptoms. Here, significant predictors added at each step are discussed, and listed in order of predictive power.

\section{Depression Symptoms}

In the first step of the model predicting depression symptoms, significant demographic and disability predictors included pain, younger age, lower energy, having multiple disabilities, being cisgender, having pre-pandemic mental health conditions, being Hispanic or Latino, lower satisfaction with ability to perform activities of daily living, and male gender. In the second step, adding pandemic-related stressors, additional significant predictors included not having basic supplies. In final step of the model, adding social factors, additional significant predictors were social isolation, disability stigma, and worries about contracting COVID-19. Each step explained a significant increase in variance and the final model explained $45.3 \%$ of the variance in participants' depression symptoms.

\section{Anxiety Symptoms}

In the first step of the model predicting anxiety symptoms, significant demographic and disability predictors were younger age, pain, having multiple disabilities, lower satisfaction with ability to perform daily activities, lower energy, having pre-pandemic mental health conditions, being cisgender, and being Hispanic or Latino. When pandemic-related stressors were added in the second step, no additional significant predictors emerged. In the final step, additional significant predictors include social isolation, disability stigma, and worries about contracting

COVID-19. Each step explained a significant increase in variance and the final model explained $40.8 \%$ of the variance in participants' anxiety symptoms.

\section{Discussion}


This descriptive study sought to identify demographic and psychosocial predictors of depression and anxiety symptoms among a sample of U.S. adults with disabilities during the COVID-19 pandemic. Descriptive research plays a vital role in laying the foundation for subsequent investigation and is often best deployed in the service of elevating insights into traditionally under-studied populations (e.g., Syed, 2017). Consistent with our hypotheses and prior research (Lund et al., 2020; Pettinicchio et al., 2021), social isolation, disability-related stigma, worries about contracting COVID-19, younger age, and physical pain were significant predictors of both depression and anxiety symptoms, and having a pre-pandemic mental health condition and difficulty accessing basic supplies were significantly associated with depression symptoms. Also, consistent with evidence indicating that the COVID-19 pandemic has been particularly stressful for people with disabilities with high support needs due to disruptions in formal and informal caregiving and support networks (Jesus et al., 2020), participants who identified as having multiple disabilities reported higher anxiety symptoms.

Although our sample does not allow us to make explicit comparisons to individuals without disabilities, it is important to offer our findings in the context of other work on the mental health implications of the COVID-19 pandemic. A representative panel survey of adults in the U.S. conducted in June of 2020 found that participants reported higher levels of depression, anxiety, substance abuse, and suicidal ideation than during the same time period in 2019; mental health concerns disproportionately affected young adults, racial/ethnic minorities, essential workers, unpaid adult caregivers, and people in treatment for pre-pandemic mental health conditions (Czeisler et al., 2020). We note that the proportion of participants reporting clinically significant levels of depression and anxiety symptoms in our sample was generally larger than in that reported by Czeisler and colleagues. However, percentages were similar 
between our sample and many of the subgroups reported by Czeisler and colleagues as experiencing elevated levels of mental health concerns.

Taken together, these findings highlight the importance of attending to experiences of social isolation, disability-related stigma, and worries about contracting COVID-19 when designing programs and initiatives aimed to support people with disabilities during the next phase of the pandemic. Notably, social isolation was the strongest predictor of both anxiety and depression. Virtual communities and support groups that connect people with disabilities with one another and build disability identity may be important tools for mitigating social isolation among people with disabilities, especially for young adults, who are less likely to affiliate with other disabled people than are their counterparts over 30 (Nario-Redmond \& Oleson, 2016). Importantly, community building opportunities foster disability identification, which has been shown to facilitate coping with stigma-related stress and increase psychological well-being (Bogart \& Hemmesch, 2016; Obst \& Stafurik, 2010).

In addition, educating healthcare providers about implicit and explicit forms of ableism, as well as incorporating individualized, culturally appropriate assessments that account for the experiences of people with disabilities into medical rationing policies, would help combat the pervasiveness of disability-related stigma in healthcare settings. Addressing healthcare stigma may also alleviate some of disabled peoples' fears and concerns about contracting COVID-19, which are in part fueled by worries about not being able to receive equitable medical treatment (Andrews et al., 2020). Furthermore, efforts should be made to ensure that people with disabilities who are at elevated mental health risk, such as those with multiple disabilities, prepandemic mental health conditions, or chronic pain, receive adequate services safely. Some exemplary strategies to address issues related to mental health, pain, and comorbid disabilities 
include psychotherapy via telehealth, home visits from healthcare or rehabilitation professionals, home delivery of basic supplies such as medications and groceries, and assistance with daily living activities. Finally, worries about contracting COVID-19 can be mitigated by ensuring guidelines to prevent the spread of COVID-19 are accessible and include people with disabilities. This includes ensuring guidance is accessible to screen readers, captioned, and includes ASL interpretation. Recommendations should address disability-specific issues such as sanitizing mobility equipment and communicating with Deaf and hard of hearing people while wearing masks.

The present study also yielded several surprising findings that warrant further investigation. First, contrary to prior research (Pettinicchio et al., 2021), experience of financial loss during the pandemic was not significantly associated with either depression or anxiety symptoms. One potential explanation for this null result is that our definition of financial loss was quite broad, encompassing challenges ranging from job loss to travel-related cancelations. As such, there might be significant heterogeneity in the extent of financial hardship experienced by participants who responded affirmatively to the single item we used to assess financial loss. In addition, because many people with disabilities struggled with unemployment and rely on government benefits (e.g., Social Security Supplemental Income, Social Security Disability Insurance) as primary sources of income even prior to the pandemic, they might be less susceptible to the pandemic's economic impacts. Items that specifically assess loss of income or employment would likely represent a more accurate indicator of financial hardship, thus better predicting mental health.

Second, participants in our sample who identified as transgender or gender nonbinary reported lower levels of depression and anxiety symptoms than their cisgender counterparts. 
These findings diverge from prior literature suggesting that transgender and gender nonbinary individuals are at increased risk for mental health problems due to stigmatization and marginalization, both in general and specifically during the COVID-19 pandemic (Alonzi et al., 2020; Meyer et al., 2017). It is also worth noting that transgender and gender nonbinary individuals represented a surprisingly substantial minority (10.3\%) in our sample. More research is needed to understand how the intersection between disability status and gender identity shapes the mental health of transgender and gender nonbinary people with disabilities and explore the unique sources of risk and resilience facing this population.

Lastly, it is interesting to note that there was a significant negative bivariate association between participant age and perceived social isolation, such that younger participants reported higher levels of social isolation than older participants. While this result might seem counterintuitive, both prior research and the current study have shown that younger adults tend to experience higher levels of psychological distress during the COVID-19 pandemic (Czeisler et al., 2021; O’Connor et al., 2021). It is possible that pandemic-related social isolation was particularly salient for young adults, who likely had more opportunities for interpersonal interactions through work or school prior to the pandemic. Another possibility is that younger participants in our study felt the impact of social isolation more acutely, given that many are likely at significantly higher risk for severe illness or death due to COVID-19 than their nondisabled peers.

The current investigation has several limitations. First, while the cross-sectional nature of our data offers a vital descriptive approach to understanding the experiences of people with disabilities during the COVID-19 pandemic, they do not allow for an assessment of causal conclusions. Whereas our conceptualization of disability-related stigma, social isolation, and 
worries about contracting COVID-19 as predictors of depression and anxiety symptoms is wellgrounded in the empirical literature (Lund et al., 2020; Pettinicchio et al., 2021), it is also possible that individuals with higher levels of depression and anxiety symptoms would be more inclined to report stigma-related experiences, social isolation, and worries about contracting COVID-19. Longitudinal studies that build on our descriptive foundation to assess these constructs repeatedly over time are needed to elucidate how psychosocial processes shape the mental health trajectory of people with disabilities over the course of the pandemic. Additionally, because all participants were recruited via disability-focused social media platforms and email listservs for disability organizations, the current sample was limited in its representativeness of the general population of U.S. adults with disabilities. Specifically, although our sample was diverse in terms of age, geographic locales, and gender identity, most of the participants were White and well-educated. Further, due to the online format of the survey, we likely missed participants who lacked assistive technology or were otherwise unable to access the Internet due to the nature of their disabilities or socioeconomic constraints. Lastly, we acknowledge that the instrument we chose to assess depressive symptoms (i.e., PHQ-9) might have produced inflated scores in some of our participants, given that several items (e.g., trouble concentrating, low energy) overlap with symptoms of common disabling chronic health conditions. Although we have somewhat mitigated this concern by adjusting for functional impairment in all of our analyses, future research should consider adopting depression assessments that are more appropriate for the disability and chronic illness communities, such as the PROMIS Emotional Distress-Depression and Anxiety Scales, which focuses on the affective component of depression (e.g., negative mood, decrease in positive affect; Pilkonis et al., 2011), and the Hospital Anxiety and Depression scale (Zigmond \& Snaith, 1983), which was designed not to confound with 
disabling conditions.

In sum, the present research extended prior literature by identifying disability-related stigma and social isolation, along with worries about contracting COVID-19, as important predictors of depression and anxiety among U.S. adults with disabilities during the COVID-19 pandemic. Our findings also underscore the need to attend to people with disabilities at elevated mental health risk, including those with multiple disabilities, pre-pandemic mental health conditions, and chronic pain, as rehabilitation professionals, disability organizations, and policymakers work to support people with disabilities in post-pandemic recovery and create a more equitable response to ongoing and future public health crises. 


\section{References}

Alonzi, S., La Torre, A., \& Silverstein, M. W. (2020). The psychological impact of preexisting mental and physical health conditions during the COVID-19 pandemic. Psychological trauma: theory, research, practice, and policy, $12(\mathrm{~S} 1)$, S236.

American Psychological Association. (2020, May 05). How americans with disabilities can cope with the COVID-19 crisis. Retrieved from https://www.apa.org/topics/covid19/disability-tip-sheet.

Andrews, E. E., Ayers, K. B., Brown, K. S., Dunn, D. S., \& Pilarski, C. R. (2020). No body is expendable: Medical rationing and disability justice during the COVID-19 pandemic. American Psychologist. Advance online publication. https://doi.org/10.1037/amp0000709

Bureau of Labor Statistics, U.S. Department of Labor. (2020). Effects of COVID-19 pandemic on the employment situation news release and data. Retrieved from https://www.bls.gov/covid19/effects-of-covid-19-pandemic-and-response-on-theemployment-situation-news-release.htm

Bogart, K. R., \& Hemmesch, A. R. (2016). Benefits of support conferences for parents of and people with Moebius syndrome. Stigma and Health, 1, 109-121. doi:10.1037/sah0000018

Branco, C., Ramos, M., \& Hewstone, M. (2019). The association of group-based discrimination with health and well-being: A comparison of ableism with other "isms". Journal of Social Issues, 74(3), 814-846.

Centers for Disease Control and Prevention. (2021). CDC COVID Data Tracker. Coronavirus Disease 2019 (COVID-19). Retrieved from https://covid.cdc.gov/covid-datatracker/\#cases_casesper100klast7days 
Centers for Disease Control and Prevention. (2020). CDC COVID-19 Community Survey Question Bank (DRAFT) Retrieved from https://cde.nlm.nih.gov/formView?tinyId=Kcceysolt.

Czeisler MÉ , Lane RI, Petrosky E, et al. (2020). Mental health, substance use, and suicidal ideation during the COVID-19 pandemic - United States, June 24-30, 2020. MMWR Morbidity and Mortality Weekly Report, 69, 1049-1057. DOI: http://dx.doi.org/10.15585/mmwr.mm6932alexternal icon.

Drum, C. E., Oberg, A., Cooper, K., \& Carlin, R. (2020). COVID-19 and adults with disabilities: health and health care access online survey summary report. Rockville, MD: American Association on Health and Disability.

Erickson, W., Lee, C., \& von Schrader, S. (2017). Disability statistics from the American Community Survey (ACS). Ithaca, NY: Cornell University Yang-Tan Institute (YTI). Retrieved from Cornell University Disability Statistics website: www. disabilitystatistics. org.

Hahn, E. A., DeWalt, D. A., Bode, R. K., Garcia, S. F., DeVellis, R. F., Correia, H., \& Cella, D. (2014). New English and Spanish social health measures will facilitate evaluating health determinants. Health Psychology, 33(5), 490.

Harkness, A., Behar-Zusman, V., \& Safren, S. A. (2020). Understanding the impact of COVID19 on Latino sexual minority men in a US HIV hot spot. AIDS and Behavior, 24(7), 2017-2023. https://doi.org/10.1007/s10461-020-02862-w

Hennein, R., Mew, E. J., \& Lowe, S. R. (2021). Socio-ecological predictors of mental health outcomes among healthcare workers during the COVID-19 pandemic in the United States. PloS one, 16(2), e0246602. 
Hwang, T. J., Rabheru, K., Peisah, C., Reichman, W., \& Ikeda, M. (2020). Loneliness and social isolation during the COVID-19 pandemic. International psychogeriatrics, 32(10), 1217 1220. https://doi.org/10.1017/S1041610220000988

Iob, E., Steptoe, A., \& Fancourt, D. (2020). Abuse, self-harm and suicidal ideation in the UK during the COVID-19 pandemic. The British Journal of Psychiatry, 217(4), 543-546. doi:10.1192/bjp.2020.130

Jesus, T., Bhattacharjya, S., Papadimitriou, C., Bogdanova, Y., Bentley, J., Arango-Lasprilla, J., \& Kamalakannan, S. (2021). Lockdown-related disparities experienced by people with disabilities during the first wave of the COVID-19 pandemic: Scoping review with thematic analysis. International Journal of Environmental Research and Public Health, 18(12). doi:10.3390/ijerph18126178

Kroenke, K., Spitzer, R. L., \& Williams, J. B. (2001). The PHQ-9: validity of a brief depression severity measure. Journal of general internal medicine, 16(9), 606-613.

Lund, E. M., Forber-Pratt, A. J., Wilson, C., \& Mona, L. R. (2020). The COVID-19 pandemic, stress, and trauma in the disability community: A call to action. Rehabilitation Psychology. Advance online publication. http://dx.doi.org/10.1037/rep0000368

Meyer, I. H., Brown, T. N. T., Herman, J. L., Reisner, S. L., \& Bockting, W. O. (2017). Demographic characteristics and health status of transgender adults in select US regions: Behavioral risk factor surveillance system, 2014. American Journal of Public Health, 107(4), 582-589. doi:10.2105/AJPH.2016.303648

Molina, Y., Choi, S. W., Cella, D., \& Rao, D. (2013). The stigma scale for chronic illnesses 8item version (SSCI-8): development, validation and use across neurological 
conditions. International journal of behavioral medicine, 20(3), 450-460. https://doi.org/10.1007/s12529-012-9243-4

Nario-Redmond, M. R., \& Oleson, K. C. (2016). Disability group identification and disabilityrights advocacy: Contingencies among emerging and other adults. Emerging Adulthood, 4, 207-218.Nario-Redmond, M. R. (2019). Ableism: The causes and consequences of disability prejudice. John Wiley \& Sons.

National Institute of Neurological Disorders and Stroke. (2015, March). User manual for the Quality of Life in Neurological Disorders (Neuro-QOL) Measures, Version 2.0. Retrieved from http://www.neuroqol.org.

Obst, P., \& Stafurik, J. (2010). Online we are all able bodied: Online psychological sense of community and social support found through membership of disability-specific websites promotes well-being for people living with a physical disability. Journal of Community \& Applied Social Psychology, 20, 525-531. doi:10.1002/casp.1067

O'Connor, R. C., Wetherall, K., Cleare, S., McClelland, H., Melson, A. J., Niedzwiedz, C. L., ... \& Robb, K. A. (2021). Mental health and well-being during the COVID-19 pandemic: longitudinal analyses of adults in the UK COVID-19 Mental Health \& Wellbeing study. The British Journal of Psychiatry, 218(6), 326-333. doi:10.1192/bjp.2020.212

Okoro, C. A., Hollis, N. D., Cyrus, A. C., \& Griffin-Blake, S. (2018). Prevalence of disabilities and health care access by disability status and type among adults—United States, 2016. Morbidity and Mortality Weekly Report, 67(32), 882. https://doi.org/10.15585/mmwr.mm6732a3 
Pettinicchio, D., Maroto, M., Chai, L., \& Lukk, M. (2021). Findings from an online survey on the mental health effects of COVID-19 on Canadians with disabilities and chronic health conditions. Disability and Health Journal, 101085. doi:10.1016/j.dhjo.2021.101085

Pilkonis, P. A., Choi, S. W., Reise, S. P., Stover, A. M., Riley, W. T., \& Cella, D. (2011). Item banks for measuring emotional distress from the Patient-Reported Outcomes Measurement Information System (PROMIS $\left.{ }^{\circledR}\right)$ : Depression, anxiety, and anger. Assessment, 18(3), 263-283. https://doi.org/10.1177/1073191111411667

Qiu, J., Shen, B., Zhao, M., Wang, Z., Xie, B., \& Xu, Y. (2020). A nationwide survey of psychological distress among Chinese people in the COVID-19 epidemic: Implications and policy recommendations. General Psychiatry, 33(2), 19-21. https://doi.org/10.1136/gpsych-2020-100213

Spitzer, R. L., Kroenke, K., Williams, J. B., \& Löwe, B. (2006). A brief measure for assessing generalized anxiety disorder: the GAD-7. Archives of internal medicine, 166(10), 10921097.

Syed, M. (2017). Why traditional metrics may not adequately represent ethnic minority psychology. Perspectives on Psychological Science, 12(6), 1162-1165.

Tabachnick, B. G., \& Fidell, L. S. (2007). Using multivariate statistics, $5^{\text {th }}$ ed. Needham Height, MA: Allyn \& Bacon.

Tough, H., Siegrist, J., \& Fekete, C. (2017). Social relationships, mental health and wellbeing in physical disability: a systematic review. BMC public health, 17(1), 1-18. doi:10.1186/s12889-017-4308-6 
Umucu, E., \& Lee, B. (2020). Examining the impact of COVID-19 on stress and coping strategies in individuals with disabilities and chronic conditions. Rehabilitation Psychology, 65(3), 193-198. doi:10.1037/rep0000328

Whoqol Group. (1998). Development of the World Health Organization WHOQOL-BREF quality of life assessment. Psychological medicine, 28(3), 551-558.

Zigmond, A. S., \& Snaith, R. P. (1983). The hospital anxiety and depression scale. Acta psychiatrica scandinavica, 67(6), 361-370. 


\section{Table 1}

Sample Demographic Characteristics

\begin{tabular}{|c|c|c|c|}
\hline Variable & $M(S D)$ & $n$ & $\%$ \\
\hline Age & $35(10.03)$ & & \\
\hline \multicolumn{4}{|l|}{ Gender } \\
\hline Male & & 173 & 38.8 \\
\hline Female & & 227 & 50.9 \\
\hline Male to female/trans woman & & 2 & 0.5 \\
\hline Female to male/trans man & & 5 & 1.1 \\
\hline $\begin{array}{l}\text { Gender queer, gender variant, or gender } \\
\text { nonconforming }\end{array}$ & & 34 & 7.6 \\
\hline Other & & 5 & 1.1 \\
\hline \multicolumn{4}{|l|}{ Race } \\
\hline American Indian or Alaska Native & & 29 & 6.5 \\
\hline Asian & & 7 & 1.6 \\
\hline Black & & 53 & 11.9 \\
\hline Native Hawaiian or Pacific Islander & & 5 & 1.1 \\
\hline White & & 317 & 71.2 \\
\hline Other & & 6 & 1.4 \\
\hline Multiracial & & 28 & 6.3 \\
\hline \multicolumn{4}{|l|}{ Ethnicity } \\
\hline Hispanic or Latino & & 88 & 19.7 \\
\hline \multicolumn{4}{|l|}{ Education } \\
\hline Some high school & & 20 & 4.5 \\
\hline High school diploma or GED & & 71 & 15.9 \\
\hline Some college or Associates Degree & & 107 & 24.0 \\
\hline Currently enrolled in college & & 35 & 7.8 \\
\hline 4-year college degree (BA, BS, BFA) & & 121 & 27.1 \\
\hline $\begin{array}{l}\text { Graduate or Professional Degree (MA, } \\
\text { MS, MD, JD, PhD, etc.) }\end{array}$ & & 91 & 20.4 \\
\hline \multicolumn{4}{|l|}{ Employment } \\
\hline Full time ( 40 hours per week) & & 157 & 35.2 \\
\hline Part time (less than 40 hours per week) & & 172 & 38.6 \\
\hline $\begin{array}{l}\text { Permanent or temporarily disabled and } \\
\text { NOT working }\end{array}$ & & 56 & 12.6 \\
\hline $\begin{array}{l}\text { Permanent or temporarily disabled } \\
\text { but working "off the books" }\end{array}$ & & 9 & 2.0 \\
\hline Unemployed-- Student & & 18 & 4.0 \\
\hline Unemployed-- Other & & 34 & 7.6 \\
\hline Income & $\$ 30,000(\$ 18,970)$ & & \\
\hline
\end{tabular}


COVID-19 Exposure

Had COVID-19

34

7.6

COVID-19 hospitalization

8

COVID-19 bereavement

28

6.3 


\section{Table 2}

Sample Disability Characteristics

\begin{tabular}{|c|c|c|c|}
\hline & $M(S D)$ & $n$ & $\%$ \\
\hline Years since disability onset & $17.49(13.75)$ & & \\
\hline \multicolumn{4}{|l|}{ Disability Type $e^{a}$} \\
\hline Emotional Behavioral Disorder & & 142 & 31.8 \\
\hline Other Health Impairment & & 119 & 26.7 \\
\hline Physical Disability & & 222 & 49.8 \\
\hline Hearing Loss or Deafness & & 70 & 15.7 \\
\hline Vision Loss or Blindness & & 52 & 11.7 \\
\hline Learning Disability & & 55 & 12.3 \\
\hline Intellectual Disability & & 17 & 3.8 \\
\hline Autism & & 41 & 9.2 \\
\hline Speech or Language Disability & & 16 & 3.6 \\
\hline Other & & 43 & 9.6 \\
\hline \multicolumn{4}{|l|}{ Disability Supports ${ }^{b}$} \\
\hline Personal care attendant & & 89 & 20.0 \\
\hline Direct support professional & & 102 & 22.9 \\
\hline Vocational rehabilitation & & 91 & 20.4 \\
\hline $\begin{array}{l}\text { An agency to support my living } \\
\text { and personal care needs }\end{array}$ & & 68 & 15.3 \\
\hline No services employed & & 241 & 54.0 \\
\hline Pre-existing Mental Health Condition & & 294 & 65.9 \\
\hline
\end{tabular}

a Participants were able to select more than one disability type.

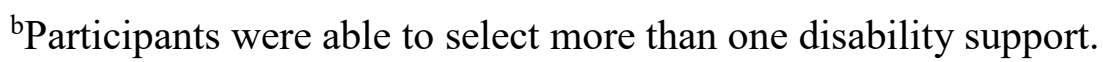




\section{Table 3}

\section{Bivariate Correlations}

\begin{tabular}{|c|c|c|c|c|c|c|c|c|c|c|c|c|c|c|c|c|c|c|c|c|}
\hline & 1 & 2 & 3 & 4 & 5 & 6 & 7 & 8 & 9 & 10 & 11 & 12 & 13 & 14 & 15 & 16 & 17 & 18 & 19 & 20 \\
\hline 1. Age & - & & & & & & & & & & & & & & & & & & & \\
\hline 2. Race $(1=\text { Non-white })^{\mathrm{a}}$ & $-0.19^{* *}$ & - & & & & & & & & & & & & & & & & & & \\
\hline 3. Ethnicity $(1=\text { Hispanic })^{\mathrm{b}}$ & -0.11 & $0.18^{* *}$ & - & & & & & & & & & & & & & & & & & \\
\hline 4. Employment $(1=\text { Employed })^{c}$ & $-0.19 * *$ & 0.07 & 0.09 & - & & & & & & & & & & & & & & & & \\
\hline 5. Education $(1=\text { Bachelor's degree or higher })^{\mathrm{d}}$ & $0.15^{* *}$ & $-0.20^{* *}$ & $-0.20 * *$ & $0.01^{*}$ & - & & & & & & & & & & & & & & & \\
\hline 6. Gender $(1=$ Female $)$ & $0.13^{* *}$ & $-0.14 * *$ & -0.09 & -0.05 & $0.23^{* *}$ & - & & & & & & & & & & & & & & \\
\hline 7. Gender ( 1 = Transgender/Non-binary $)$ & -0.01 & 0 & -0.04 & -0.08 & $0.18^{* *}$ & $-0.35^{* *}$ & - & & & & & & & & & & & & & \\
\hline 8. Number of Disabilities $(1=\text { Multiple impairments })^{e}$ & 0.03 & -0.05 & -0.05 & $-0.117^{*}$ & $0.23 * *$ & 0.03 & $0.31^{* *}$ & - & & & & & & & & & & & & \\
\hline 9. Pre-pandemic mental health conditions & 0.08 & -0.01 & $-0.13 * *$ & $-0.15^{* *}$ & $0.20^{* *}$ & 0.07 & $0.20 * *$ & $0.34 * *$ & - & & & & & & & & & & & \\
\hline 10. Physical pain & 0.01 & 0.09 & 0.04 & 0.03 & -0.05 & -0.06 & $0.12 * *$ & $0.20 * *$ & 0.07 & - & & & & & & & & & & \\
\hline 11. Daily energy & 0.03 & -0.02 & -0.03 & $0.15^{* *}$ & $0.12 *$ & -0.03 & $-0.15^{* *}$ & $-0.16^{* *}$ & $-0.14^{* *}$ & $-0.22 * *$ & - & & & & & & & & & \\
\hline 12. Ability to perform daily tasks & -0.04 & 0.06 & 0.06 & $0.30^{* *}$ & 0.04 & -0.09 & $-0.16^{* *}$ & $-.10^{*}$ & $-0.17^{* *}$ & $-0.10^{*}$ & $0.46^{* *}$ & - & & & & & & & & \\
\hline 13. Personal financial loss & -0.06 & -0.04 & -0.05 & $0.11^{*}$ & $0.13^{* *}$ & 0.09 & 0.07 & -0.02 & 0.05 & -0.04 & -0.04 & $-0.10^{*}$ & - & & & & & & & \\
\hline 14. Lack of basic supplies & -0.04 & $0.14 * *$ & 0.03 & -0.05 & $-0.12 *$ & -0.02 & 0.09 & $0.23^{* *}$ & 0.03 & $0.19^{* *}$ & $-0.12 *$ & -0.05 & 0.06 & - & & & & & & \\
\hline 15. COVID-19 worries & -0.02 & $-0.12^{* *}$ & -0.06 & 0.01 & -0.01 & 0.09 & $-0.10^{*}$ & 0.03 & -0.05 & 0.09 & -0.02 & -0.07 & 0.05 & 0.06 & - & & & & & \\
\hline 16. COVID-19 status ${ }^{\mathrm{f}}$ & -0.01 & 0.06 & 0.07 & -0.07 & 0 & -0.04 & $0.15^{* *}$ & $0.12^{*}$ & 0.08 & 0.09 & -0.08 & -0.06 & 0.06 & 0.01 & -0.07 & - & & & & \\
\hline 17. COVID-19 bereavement ${ }^{\mathrm{g}}$ & 0.01 & $0.14 * *$ & 0.03 & 0.05 & -0.06 & 0.07 & -0.09 & $-0.10^{*}$ & -0.03 & -0.03 & -0.03 & 0.04 & 0.07 & -0.09 & 0.09 & -0.04 & - & & & \\
\hline 18. Social isolation & $-0.13^{* *}$ & 0 & 0.03 & $-0.01 *$ & 0.02 & -0.04 & $0.21 * *$ & $0.31^{* *}$ & $0.21^{* *}$ & $0.25^{* *}$ & $-0.20 * *$ & $-0.18^{* *}$ & 0.08 & $0.15^{* *}$ & 0.01 & $0.12^{* *}$ & -0.06 & - & & \\
\hline 19. Disability stigma & -0.09 & 0.09 & $0.12^{*}$ & $-.10^{*}$ & -0.08 & $-0.01 *$ & 0.06 & $0.23^{* *}$ & $0.13^{* *}$ & $0.34^{* *}$ & $-0.24 * *$ & $-0.14^{* *}$ & -0.01 & $0.16^{* *}$ & -0.05 & 0.04 & -0.03 & $0.56^{* *}$ & - & \\
\hline 20. PHQ-9 & $-0.22 * *$ & $0.17 * *$ & $0.15^{* *}$ & -0.02 & $-0.10^{*}$ & -0.05 & 0.03 & $0.25^{* *}$ & $0.18^{* *}$ & $0.40^{* *}$ & $-0.34 * *$ & $-0.21^{* *}$ & 0.06 & $0.25^{* *}$ & $0.11^{*}$ & 0.09 & 0.04 & $0.46^{* *}$ & $0.46^{* *}$ & - \\
\hline 21. GAD- $7^{j}$ & $-0.25^{* *}$ & $0.11^{*}$ & $0.11^{*}$ & -0.03 & -0.08 & -0.01 & 0.05 & $0.27^{* *}$ & $0.17^{* *}$ & $0.35^{* *}$ & $-0.27^{* *}$ & $-0.21 * *$ & 0.08 & $0.20^{* *}$ & $0.12^{* *}$ & 0.05 & 0.06 & $0.47 * *$ & $0.44 * *$ & $0.81^{*}$ \\
\hline
\end{tabular}

$*$ = Correlation is significant at the .05 level, $* *=$ Correlation significant at the .01 level.

${ }^{\mathrm{a}} 0=$ White, $1=$ Non-white.

${ }^{\mathrm{b}} 0=$ Non-Hispanic, $1=$ Hispanic.

${ }^{\mathrm{c}} 0=$ Unemployed, $1=$ Employed.

${ }^{\mathrm{d}} 0=$ less than a bachelor's degree, $1=$ Bachelor's degree or higher. 
${ }^{\mathrm{e}} 0=$ Single disability, $1=$ Multiple disabilities .

${ }^{\mathrm{f}} 0=$ No, $1=$ Yes.

${ }^{\mathrm{g}} 0=$ No, $1=$ Yes/Probably

${ }^{\mathrm{h}} 0=$ No, $1=$ Yes.

${ }^{\text {i }}$ Patient Health Questionnaire - 9.

${ }^{\mathrm{j}}$ Generalized Anxiety Disorder - 7 


\section{Table 4}

Hierarchical Regressions Predicting Depression and Anxiety

\begin{tabular}{|c|c|c|c|c|}
\hline \multirow[b]{3}{*}{ Predictor Variable } & \multicolumn{4}{|c|}{ Outcome variable } \\
\hline & \multicolumn{2}{|c|}{ Depression } & \multicolumn{2}{|c|}{ Anxiety } \\
\hline & $b(\mathrm{SE})$ & $\mathrm{B}$ & $b(\mathrm{SE})$ & $\beta$ \\
\hline \multicolumn{5}{|l|}{ Step 1: Demographic covariates } \\
\hline Physical pain & $1.70(0.23)$ & $0.30 * *$ & $1.25(0.19)$ & $0.27 * *$ \\
\hline Energy & $-1.03(0.25)$ & $-0.19 * *$ & $-0.49(0.21)$ & $-0.11 *$ \\
\hline Ability to perform daily tasks & $-0.55(0.25)$ & $-0.10^{*}$ & $-0.52(0.21)$ & $-0.12 *$ \\
\hline Age & $-0.12(0.02)$ & $-0.21 * *$ & $-0.12(0.02)$ & $-0.25 * *$ \\
\hline Pre-pandemic mental health conditions & $1.59(0.54)$ & $0.13 * *$ & $1.10(0.45)$ & $0.11 *$ \\
\hline Ethnicity & $1.68(0.61)$ & $0.11 * *$ & $0.99(0.51)$ & 0.08 \\
\hline Employment & $0.02(0.61)$ & 0.00 & $-0.15(0.51)$ & -0.01 \\
\hline Race & $1.05(0.54)$ & 0.08 & $0.30(0.46)$ & 0.03 \\
\hline Gender $($ Female $=1)$ & $-0.66(0.54)$ & -0.06 & $-0.04(0.45)$ & 0.00 \\
\hline Gender $($ Transgender/Non-binary $=1)$ & $-2.88(0.91)$ & $-0.15 * *$ & $-1.55(0.77)$ & $-0.10^{*}$ \\
\hline Education & $-0.26(0.53)$ & -0.02 & $-0.34(0.46)$ & -0.04 \\
\hline Number of disabilities & $2.10(0.53)$ & $0.18 * *$ & $1.93(0.45)$ & $0.20 * *$ \\
\hline \multicolumn{5}{|l|}{ Step 2: Pandemic related stressors } \\
\hline Physical pain & $1.59(0.23)$ & $0.29 * *$ & $1.20(0.19)$ & $0.27 * *$ \\
\hline Energy & $-0.99(0.25)$ & $-0.18 * *$ & $-0.47(0.21)$ & $-0.10^{*}$ \\
\hline Ability to perform daily tasks & $-0.51(0.25)$ & $-0.09 *$ & $-0.48(0.21)$ & $-0.11 *$ \\
\hline Age & $-0.12(0.02)$ & $-0.2 * *$ & $-0.12(0.02)$ & $-0.25 * *$ \\
\hline Pre-pandemic mental health conditions & $1.67(0.53)$ & $0.13 * *$ & $1.15(0.45)$ & $0.11 *$ \\
\hline Ethnicity & $1.74(0.60)$ & $0.12 * *$ & $1.08(0.51)$ & $0.09 *$ \\
\hline Employment & $-0.06(0.61)$ & 0.00 & $-0.31(0.52)$ & -0.03 \\
\hline Race & $0.89(0.55)$ & 0.07 & $0.23(0.47)$ & 0.02 \\
\hline
\end{tabular}


Gender $($ Female $=1)$

Gender (Transgender/Non-binary $=1)$

Education

Number of disabilities

Worries about COVID-19

Had COVID-19

COVID-19 bereavement

Personal financial loss

Lack of basic supplies

$\Delta \mathrm{R}^{2}$

Total $\mathrm{R}^{2}$

Step 3: Social factors

Physical pain

Energy

Ability to perform daily tasks

Age

Pre-pandemic mental health conditions

Ethnicity

Employment

Race

Gender $($ Female $=1)$

Gender (Transgender/Non-binary $=1)$

Education

Number of disabilities

Worries about COVID-19

Had COVID-19

COVID-19 bereavement

Personal financial loss

$\begin{array}{cccc}-0.85(0.53) & -0.07 & -0.21(0.45) & -0.02 \\ -2.95(0.92) & -0.15^{* *} & -1.50(0.77) & -0.09 \\ -0.08(0.54) & -0.01 & -0.28(0.46) & -0.03 \\ 1.78(0.55) & 0.15^{* *} & 1.84(0.46) & 0.19^{* *} \\ 0.20(0.13) & 0.06 & 0.18(0.11) & 0.07 \\ 0.54(0.89) & 0.02 & -0.39(0.75) & -0.02 \\ 1.12(0.98) & 0.05 & 1.40(0.83) & 0.07 \\ 0.55(0.49) & 0.05 & 0.67(0.41) & 0.07 \\ 1.47(0.52) & 0.12^{* *} & 0.67(0.44) & 0.07 \\ & 0.02 & & 0.02 \\ & 0.37 & & 0.32\end{array}$

$\begin{array}{cccc}1.14(0.22) & 0.21^{* *} & 0.82(0.19) & 0.18^{* *} \\ -0.84(0.23) & -0.15^{* *} & -0.34(0.20) & -0.08 \\ -0.38(0.23) & -0.07 & -0.37(0.20) & -0.08 \\ -0.09(0.02) & -0.15^{* *} & -0.09(0.02) & -0.20^{* *} \\ 1.25(0.50) & 0.10^{*} & 0.79(0.42) & 0.08 \\ 1.36(0.57) & 0.09^{*} & 0.76(0.48) & 0.06 \\ 0.34(0.57) & 0.02 & 0.03(0.48) & 0 \\ 1.00(0.52) & 0.08 & 0.32(0.44) & 0.03 \\ -0.64(0.50) & -0.05 & -0.04(0.43) & 0 \\ -2.93(0.87) & -0.15 * * & -1.50(0.73) & -0.09 * \\ -0.03(0.51) & 0.00 & -0.24(0.43) & -0.02 \\ 0.97(0.52) & 0.08 & 1.15(0.44) & 0.12^{* *} \\ 0.26(0.12) & 0.08^{*} & 0.22(0.10) & 0.08^{*} \\ 0.43(0.83) & 0.02 & -0.50(0.70) & -0.03 \\ 1.19(0.92) & 0.05 & 1.46(0.77) & 0.07 \\ 0.32(0.46) & 0.03 & 0.46(0.39) & 0.05\end{array}$


Lack of basic supplies

Disability stigma

Social isolation

$\Delta \mathrm{R}^{2}$

Total $\mathrm{R}^{2}$

Note $\mathrm{SE}=$ standard error.

${ }^{*} \mathrm{p}<.05,{ }^{* *} \mathrm{p}<.01$.

$\begin{array}{cccc}1.27(0.49) & 0.10^{* *} & 0.50(0.41) & 0.05 \\ 0.17(0.05) & 0.15^{* *} & 0.14(0.05) & 0.15^{* *} \\ 0.18(0.04) & 0.22 * * & 0.15(0.03) & 0.24 * * \\ & 0.08 & & 0.09 \\ & 0.45 & & 0.41\end{array}$

